\title{
SECCSL: Security Concurrent Separation Logic
}

\author{
Gidon Ernst ${ }^{1(\bowtie)}$ and Toby Murray ${ }^{2}$ \\ 1 LMU Munich, Munich, Germany \\ gidon.ernst@imu.de \\ 2 University of Melbourne, Melbourne, Australia \\ toby.murray@unimelb.edu.au
}

\begin{abstract}
We present SECCSL, a concurrent separation logic for proving expressive, data-dependent information flow security properties of low-level programs. SECCSL is considerably more expressive, while being simpler, than recent compositional information flow logics that cannot reason about pointers, arrays etc. To capture security concerns, SECCSL adopts a relational semantics for its assertions. At the same time it inherits the structure of traditional concurrent separation logics; thus SECCSL reasoning can be automated via symbolic execution. We demonstrate this by implementing SECC, an automatic verifier for a subset of the C programming language, which we apply to a range of benchmarks.
\end{abstract}

\section{Introduction}

Software verification successes abound, whether via interactive proof or via automatic program verifiers. While the former has yielded individual, deeply verified software artifacts $[21,24,25]$ primarily by researchers, the latter appears to be having a growing impact on industrial software engineering [11,36,39].

At the same time, recent work has heralded major advancements in program logics for reasoning about secure information flow $[23,33,34]$ - i.e. whether programs properly protect their secrets - yielding the first general program logics and proofs of information flow security for non-trivial concurrent programs [34]. Yet so far, such logics have remained confined to interactive proof assistants, making them practically inaccessible to industrial developers.

This is not especially surprising. The COVERN logic [34], for example, pays for its generality with regard to expressive security policies, in terms of complexity. Worse, these logics reason only over very simple toy programming languages, which even lack support for pointers, arrays, and structures. Their complexity, we argue, hinders proof automation and makes scaling up these logics to real-world languages impractical. How, therefore, can we leverage the power of existing automatic deductive verification approaches for security proofs?

In this paper we present Security Concurrent Separation Logic (SECCSL), which achieves an unprecedented combination of simplicity, power, and ease of 
automation by capturing core concepts such as data-dependent variable sensitivity [27,31,50], and shared invariants on sensitive memory [34] in the familiar style of Concurrent Separation Logic (CSL) [38], as exemplified in Sect. 2.

Prior work $[14,20]$ has noted the promise of separation logic for reasoning about information flow yet, to date, that promise remains unrealised. Indeed, the only two prior encodings of information flow concepts into separation logics which we are aware of have overlooked crucial features like concurrency [14], and lack the ability to separately specify the sensitivity of values and memory locations as we explain in Sect. 2. The logic in [20] lacks soundness arguments altogether while [14] fail to satisfy basic properties needed for automation (see the discussion following Proposition 1).

Designing a logic with the right combination of features, with the right semantics, is therefore non-trivial. To manage this, SECCSL assertions have a relational interpretation [6,49] over a standard heap model (Sect.3). This allows one to canonically encode information flow concepts while maintaining the approach and structure of traditional CSL proofs. To do so we adapt existing proof techniques for the soundness of CSL [46] into a compositional information flow security property (Sect.4) that, like SECCSL itself, is simple and powerful. We have mechanized the soundness of SECCSL in Isabelle/HOL [37].

To demonstrate SECCSL's ease of use and capacity for automation, we implemented the prototype tool SECC (Sect.5). We target C because it dominates low-level security-critical code. SECC automates SECCSL reasoning via symbolic execution, in the style of contemporary Separation Logic program verifiers like VeriFast [22], Viper [30], and Infer [10]. SECC correctly analyzes well-known benchmark problems (collected in [17]) within a few milliseconds; and we verify a variant of the CDDC case study [5] from the COVERN project. Our Isabelle theories, the open source prototype tool SECC, and examples are available online at https://covern.org/secc [18].

\section{An Overview of SECCSL}

\subsection{Specifying Information Flow Control in SECCSL}

Consider the program in Fig. 1. It maintains a global pointer rec to a shared record, protected by the lock mutex. The is_classified field of the record identifies the confidentiality of the record's data: when is_classified is true, the value stored in the data field is confidential, and otherwise it is safe to release publicly. The left thread outputs the data in the record whenever it is public by writing to the (memory mapped) output device register pointer OUTPUT_REG (here also protected by mutex). The right thread updates the record, ensuring its content is not confidential, here by clearing its data.

Suppose assigning a value $d$ to the OUTPUT_REG register causes $d$ to be outputted to a publicly-visible location. Reasoning, then, that the example is secure requires capturing that (1) the data field of the record pointed to by rec is confidential precisely when the record's is_classified field says it is, and (2) data 


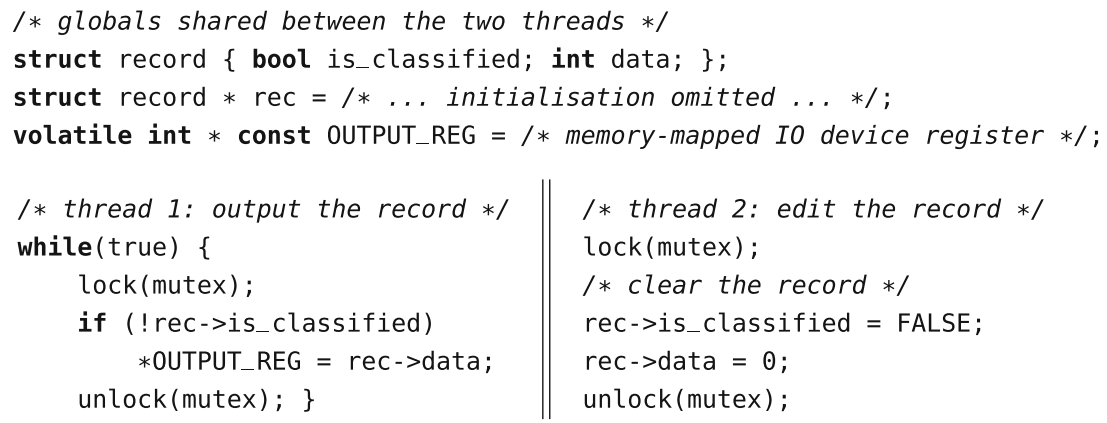

Fig. 1. Example of concurrent information flow.

sink OUTPUT_REG should never have confidential data written to it. Therefore the example only ever writes non-confidential data into OUTPUT_REG.

Condition (1) specifies the sensitivity of a data value in memory, whereas condition (2) specifies the sensitivity of the data that a memory location (i.e. data sink) is permitted to hold. Prior security separation logics $[14,20]$ reason only about value-sensitivity condition (1) but, as we explain below, both are needed. Like those prior logics, in SECCSL one specifies the sensitivity of the value denoted by an expression $e$ via a security label $\ell$ : the assertion $e:: \ell$ means that the sensitivity of the value denoted by expression $e$ is at most $\ell$. Security labels are drawn from a lattice with top element high (denoting the most confidential information), bottom element low (denoting public information), and ordered via $\sqsubseteq: \ell \sqsubseteq \ell^{\prime}$ means that information labelled with $\ell^{\prime}$ is at least as sensitive as that labelled by $\ell$. Using this style of assertion, in conjunction with standard separation logic connectives (explained below), condition (1) can be specified as:

$$
\exists c d . \operatorname{rec} \mapsto(c, d) \wedge c:: \text { low } \wedge d::(c \text { ? high : low })
$$

Separation logic's points-to predicate $e \mapsto e^{\prime}$ means the memory location denoted by expression $e$ holds the value denoted by $e^{\prime}$. Thus (1) can be read as saying that the rec pointer points to a pair of values $(c, d)$. The first $c$ (the value of the is_classified field) is public. The sensitivity of the second $d$ (the value of the data field) is given by the value of the first $c$ : it is high when $c$ is true and is low otherwise. SECCSL integrates such reasoning about valuedependent sensitivity $[27,31,50]$ neatly with functional properties of low-level data structures, which we think is more natural and straightforward than the approach of $[34,35]$ that keeps the two concerns separate.

Value-sensitivity assertion $e:: \ell$ is a judgement on the maximum sensitivity of the data source(s) from which $e$ has been derived. Location-sensitivity assertions, on the other hand, are used to specify security policies on data sinks like OUTPUT_REG. These assertions augment the separation logic points-to predicate 
with a security label $\ell$, and are used to specify which parts of the memory are observable to the attacker (and so must never contain sensitive information): $e \stackrel{\ell}{\mapsto} e^{\prime}$ means that the value denoted by the expression $e^{\prime}$ is present in memory at the location denoted by $e$, and additionally that at all times the sensitivity of the value stored in that locations is never allowed to exceed $\ell$. Thus in SECCSL, $e \mapsto e^{\prime}$ abbreviates $e \stackrel{\text { high }}{\longmapsto} e^{\prime}$. In Fig. 1, that OUTPUT_REG is publicly-observable can be specified as:

$$
\exists v . \text { OUTPUT_REG } \stackrel{\text { low }}{\longmapsto} v
$$

\subsection{Reasoning in SECCSL}

SECCSL judgements have the form:

$$
\ell_{A} \vdash\{P\} c\{Q\}
$$

Here $\ell_{A}$ is the attacker security level, $c$ is the (concurrent) program command being executed, and $P$ and $Q$ are the program's pre- resp. postcondition. Judgement (3) means that if the program $c$ begins in a state satisfying its precondition $P$ then, when it terminates, the final state will satisfy its postcondition $Q$. Analogously to [44] the program is guaranteed to be memory safe. We defer a description of $\ell_{A}$ and the implied security property to Sect. 2.3.

As with traditional CSLs, SECCSL is geared towards reasoning over sharedmemory programs that use lock-based synchronisation. Each lock $l$ has an associated invariant $\operatorname{inv}(l)$, which is simply a predicate, like $P$ or $Q$ in (3), that describes the shared memory that the lock protects. In Fig. 1, where the lock mutex protects the shared pointer rec and OUTPUT_REG, the associated invariant $\operatorname{inv}($ mutex) is simply the conjunction of (1) and (2).

$$
(\exists c d . \operatorname{rec} \mapsto(c, d) \wedge c:: \text { low } \wedge d::(c \text { ? high : low })) \star(\exists v \text {. OUTPUT_REG } \stackrel{\text { low }}{\longmapsto} v)
$$

Separating conjunction $P \star Q$ asserts that the assertions $P$ and $Q$ both hold and, additionally, that the memory locations referenced by $P$ and $Q$ respectively do not overlap. Thus SECCSL invariants, like SECCSL assertions, describe together both functional properties (e.g. rec is a valid pointer) and security concerns (e.g. the OUTPUT_REG location is publicly visible) of the shared state.

When acquiring a lock one gets to assume that the lock's invariant holds [38]. Subsequently, when releasing the lock one must prove that the invariant has been re-established. For example, when reasoning about the code of the left-thread in Fig. 1, upon acquiring the mutex, SECCSL adds formula (4) to the intermediate assertion, which allows proving that the loop body is secure. When reasoning about the right thread, one must prove that the invariant has been re-established when it releases the mutex. This is the reason e.g. that the right thread must clear the data field after setting is_classified to false. 
Reasoning in SECCSL proceeds forward over the program text according to the rules in Fig. 4. When execution forks, as in Fig. 1, one reasons over each thread individually. For Fig. 1, SECCSL requires proving that the guard of the if-condition is low, i.e. that the program is not branching on a secret (rule IF in Fig. 4), which would correspond to a timing channel, see Sect. 2.3 below. This follows from the part $c$ :: low of invariant (4). Secondly, after the write to OUTPUT_REG, SECCSL requires that the expression that is being written to the location OUTPUT_REG has sensitivity low (rule STORE in Fig. 4). This follows from $d::(c$ ? high : low) in the invariant, which simplifies to $d::$ high given the guard $c \equiv$ true of the if-statement. Finally, when the right thread releases mutex, invariant (4) holds for the updated contents of rec (rule UNLOCK in Fig. 4).

\subsection{Security Intuition and Informal Security Property}

But what does security mean in SECCSL? Indeed, the SECCSL a judgement $\ell_{A} \vdash\{P\} c\{Q\}$ additionally implies that the program $c$ does not leak any sensitive information during its execution to potential attackers.

The attacker security level $\ell_{A}$ in (3) represents an upper bound on the parts of the program's memory that a potential, passive attacker is assumed to be able to observe before, during, and after the program's execution. Intuitively this encompasses all memory locations whose sensitivity is $\sqsubseteq \ell_{A}$. Which memory locations have sensitivity $\sqsubseteq \ell_{A}$ is defined by the location-sensitivity assertions in the precondition $P$ and the lock invariants: A memory location $l o c$ is visible to the $\ell_{A}$ attacker iff $P$ or a lock invariant contains some $e \stackrel{e_{l}}{\longmapsto} e^{\prime}$ and in the program's initial state $e$ evaluates to $l o c$ and $e_{l}$ evaluates to some label $\ell$ such that $\ell \sqsubseteq \ell_{A}$ (see Fig. 3 ).

Which data is sensitive and should not be leaked to the $\ell_{A}$ attacker is defined by the value-sensitivity assertions in $P$ and the lock invariants: an expression $e$ is sensitive when $P$ or a lock invariant contains some $e:: e_{l}$ and in the program's initial state $e_{l}$ evaluates to some $\ell$ with $\ell \nsubseteq \ell_{A}$. Security, then, requires that in all intermediate states of the program's execution no sensitive data (as defined by value-sensitivity assertions) can be inferred via the attacker-observable memory (as defined by location-sensitivity assertions).

SECCSL proves a compositional security property that formalises this intuition (see Definition 3). Since the property needs to be compositional with regards to concurrent execution, the resulting security property is timing sensitive, meaning that not only must the program never reveal sensitive data into attacker-observable memory locations but the times at which it updates these memory locations cannot depend on sensitive data. It is well-known that timinginsensitive security properties are not compositional under standard scheduling models $[34,48]$. For this reason SECCSL forbids programs from branching on sensitive values. We believe that this restriction could in principle be relaxed in the future via established techniques [28,29].

SECCSL's top-level soundness (Sect.4) formalises the above intuitive definition of security in the style of traditional noninterference [19] that compares two program executions with respect to the observations that can be made by 
an attacker. SECCSL adopts a relational interpretation for the assertions $P$ and $Q$, and the lock invariants, in which they are evaluated against pairs of execution states. This relational semantics directly expresses the comparison needed for noninterference. As a result, most of the complexities related to SECCSL's soundness are confined to the semantic level, whereas the calculus retains its similarity to standard separation logic and hence its simplicity.

Under this relational semantics (see Fig. 2 in Sect. 3), when a pair of states satisfies an assertion $P$, it implies that the two states agree on the values of all non-sensitive expressions as defined by $P$ (Lemma 1). Noninterference is then stated as Theorem 2: Program $c$ with precondition $P$ is secure against the $\ell_{A^{-}}$ attacker if, whenever executed twice from two initial states jointly satisfying $P$ and the lock invariants (and so agreeing on the values of all data assumed to be initially observable to the $\ell_{A}$ attacker), in all intermediate pairs of states arrived at after running each execution for the same number of steps, the resulting states again agree at that initially $\ell_{A}$-visible memory. This definition is timing sensitive as it compares executions that have the same number of steps.

\section{The Logic SECCSL}

\subsection{Assertions}

Pure expressions $e$ that do not depend on the heap are composed of variables $x$, function applications, equations, and conditional expressions. Pure relational formulas $\rho$ comprise boolean expressions $\phi$, value sensitivity $e:: e_{l}$, and relational implication $\Rightarrow$ (wlog. covering relational $\neg, \wedge, \vee$ ). We assume a standard firstorder many sorted typing discipline (not elaborated).

$$
e::=x\left|f\left(e_{1}, \ldots, e_{n}\right)\right| e_{1}=e_{2}\left|\phi ? e_{1}: e_{2} \quad \rho::=\phi\right| e:: e_{l} \mid \rho_{1} \Rightarrow \rho_{2}
$$

We postulate that the logical signature contains a sort Label, corresponding to the security lattice, with constants low, high: Label and a binary predicate symbol $\sqsubseteq$ : Label $\times$ Label $\rightarrow$ Bool, whose interpretation satisfies the lattice axioms.

SECCSL's assertions $P, Q$ may additionally refer to the heap and thus include the empty heap description, labelled points-to predicates (heap location sensitivity assertions), assertions guarded by (pure) conditionals, ordinary overlapping conjunction as well as separating conjunction, and existential quantification.

$$
P::=\rho \mid \text { emp }\left|e_{p} \stackrel{e_{l}}{\longmapsto} e_{v}\right|(\phi ? P: Q)|P \wedge Q| P \star Q \mid \exists x . P
$$

Disjunction, negation, and implication are excluded because they cause issues for describing the set of $\ell$-visible heap location to the $\ell$-attacker, similarly to the problem of defining heap footprints for non-precise assertions [26,40,41]. These connectives can still occur between pure and relational expressions.

The standard expression semantics $\llbracket e \rrbracket_{s}$ evaluates $e$ over a store $s$, which assigns values to variables $x$ as $s(x)$. The interpretation $f^{\mathcal{A}}$ of a function symbol $f$ is a function, given statically by a logical structure $\mathcal{A}$. Specifically, $\complement^{\mathcal{A}}$ is the semantic ordering of the security lattice. We write $s \models \phi$ if $\llbracket \phi \rrbracket_{s}=$ true. 
The relational semantics of assertions, written $(s, h),\left(s^{\prime}, h^{\prime}\right) \models_{\ell} P$, is defined in Fig. 2 over two states $(s, h)$ and $\left(s^{\prime}, h^{\prime}\right)$ each consisting of a store and a heap. The semantics is defined against the attacker security level $\ell$ (called $\ell_{A}$ in Sect. 2.3). Stores $s$ and $s^{\prime}$ are related via $e:: e_{l}$. We require the expression $e_{l}$ denoting the sensitivity to coincide on $s$ and $s^{\prime}$ and whenever $\llbracket e_{l} \rrbracket_{s} \sqsubseteq^{\mathcal{A}} \ell$ holds, $e$ must evaluate to the same value both states, (7). Heaps are related by $(s, h),\left(s^{\prime}, h^{\prime}\right) \models_{\ell} e_{p} \stackrel{e_{l}}{\longmapsto} e_{v}$, which similarly ensures that the two heap fragments are identical $h=h^{\prime}$ when $e_{l}$ says so, (9). Conditional assertions $\phi ? P: Q$ evaluate to $P$ when $\phi$ holds (relationally), and to $Q$ otherwise. The separating conjunction splits both heaps independently, (12). Similarly, the existential quantifier picks two values $v$ and $v^{\prime},(13)$. Whether parts of the split resp. these two values actually agree will depend on other assertions made.

$$
\begin{aligned}
& \text { Using the abbreviation } s, h \models e_{p} \mapsto e_{v} \Longleftrightarrow h=\left\{\llbracket e_{p} \rrbracket_{s} \mapsto \llbracket e_{v} \rrbracket_{s}\right\} \\
& (s, h),\left(s^{\prime}, h^{\prime}\right) \models_{\ell} \text { emp } \Longleftrightarrow h=h^{\prime}=\varnothing \\
& (s, h),\left(s^{\prime}, h^{\prime}\right) \models_{\ell} \phi \Longleftrightarrow s \models \phi \text { and } s^{\prime} \models \phi \\
& (s, h),\left(s^{\prime}, h^{\prime}\right) \models_{\ell} e:: e_{l} \\
& \Longleftrightarrow \llbracket e_{l} \rrbracket_{s}=\llbracket e_{l} \rrbracket_{s^{\prime}} \text { and }\left(\llbracket e_{l} \rrbracket_{s} \sqsubseteq^{\mathcal{A}} \ell \Longrightarrow \llbracket e \rrbracket_{s}=\llbracket e \rrbracket_{s^{\prime}}\right) \\
& (s, h),\left(s^{\prime}, h^{\prime}\right) \models_{\ell} \rho_{1} \Rightarrow \rho_{2} \\
& \Longleftrightarrow(s, h),\left(s^{\prime}, h^{\prime}\right) \models_{\ell} \rho_{1} \text { implies }(s, h),\left(s^{\prime}, h^{\prime}\right) \models_{\ell} \rho_{2} \\
& (s, h),\left(s^{\prime}, h^{\prime}\right) \models_{\ell} e_{p} \stackrel{e_{l}}{\longmapsto} e_{v} \\
& \Longleftrightarrow s, h \models e_{p} \mapsto e_{v} \text { and } s^{\prime}, h^{\prime} \models e_{p} \mapsto e_{v} \text { and }(s, h),\left(s^{\prime}, h^{\prime}\right) \models e_{p}:: e_{l} \wedge e_{v}:: e_{l} \\
& (s, h),\left(s^{\prime}, h^{\prime}\right) \models_{\ell}(\phi ? P: Q) \\
& \Longleftrightarrow \begin{cases}(s, h),\left(s^{\prime}, h^{\prime}\right) \models_{\ell} P, & \text { if } s \models \phi \text { and } s^{\prime} \models \phi \\
(s, h),\left(s^{\prime}, h^{\prime}\right) \models_{\ell} Q, & \text { otherwise }\end{cases} \\
& (s, h),\left(s^{\prime}, h^{\prime}\right) \models_{\ell} P \wedge Q \\
& \Longleftrightarrow(s, h),\left(s^{\prime}, h^{\prime}\right) \models_{\ell} P \text { and }(s, h),\left(s^{\prime}, h^{\prime}\right) \models_{\ell} Q \\
& (s, h),\left(s^{\prime}, h^{\prime}\right) \models_{\ell} P \star Q \\
& \Longleftrightarrow \text { there are disjoint sub-heaps } h_{1}, h_{2} \text { and } h_{1}^{\prime}, h_{2}^{\prime} \\
& \text { with } h=h_{1} \uplus h_{2} \text { and } h^{\prime}=h_{1}^{\prime} \uplus h_{2}^{\prime} \\
& \text { such that }\left(s, h_{1}\right),\left(s^{\prime}, h_{1}^{\prime}\right) \models_{\ell} P_{1} \text { and }\left(s, h_{2}\right),\left(s^{\prime}, h_{2}^{\prime}\right) \models_{\ell} P_{2} \\
& (s, h),\left(s^{\prime}, h^{\prime}\right) \models_{\ell} \exists x . P \\
& \Longleftrightarrow \text { there are values } v, v^{\prime} \text { such that }(s(x:=v), h),\left(s^{\prime}\left(x:=v^{\prime}\right), h^{\prime}\right) \models P
\end{aligned}
$$

Fig. 2. Relational semantics of assertions.

To capture strong security properties, we require a declarative specification of which heap locations are considered visible to the $\ell$-attacker, when assertion $P$ 


$$
\begin{aligned}
\operatorname{lows}_{\ell}(\rho, s) & =\emptyset, \quad \text { notably } \operatorname{lows}_{\ell}\left(e:: e_{l}, s\right)=\emptyset \\
\operatorname{lows}_{\ell}(P \star Q, s) & =\operatorname{lows}_{\ell}(P \wedge Q, s)=\operatorname{lows}_{\ell}(P, s) \cup \operatorname{lows}_{\ell}(Q, s) \\
\operatorname{lows}_{\ell}\left(e_{p} \stackrel{e_{l}}{\longmapsto} e_{v}, s\right) & = \begin{cases}\left\{\llbracket e_{p} \rrbracket_{s}\right\}, & \llbracket e_{l} \rrbracket_{s} \sqsubseteq \mathcal{A} \ell \\
\emptyset, & \text { otherwise }\end{cases} \\
\operatorname{lows}_{\ell}(\phi ? P: Q, s) & = \begin{cases}\operatorname{lows}_{\ell}(P, s), & s \models \phi \\
\operatorname{lows}_{\ell}(Q, s), & \text { otherwise }\end{cases} \\
\operatorname{lows}_{\ell}(\exists x . P, s) & = \begin{cases}\operatorname{lows}_{\ell}(P, s), & \forall v . \operatorname{lows}_{\ell}(P, s)=\operatorname{lows}_{\ell}(P, s(x \mapsto v)) \\
\emptyset, & \text { otherwise }\end{cases}
\end{aligned}
$$

Fig. 3. Low locations of an assertion.

holds in some (initial) state (see Sect. 2.3). We define this set in Fig. 3, denoted $\operatorname{lows}_{\ell}(P, s)$ for initial store $s$. Note that, by design, the definition does not give a useful result for an existential like $\exists p v . p \stackrel{\text { low }}{\longmapsto} v$. This mirrors the usual difficulty of defining footprints for non-precise separation logic assertions [26,40,41]. This restriction is not an issue in practice, as location sensitivity assertions $e_{p} \stackrel{e_{l}}{\longmapsto} e_{v}$ are intended to describe the static regions of memory (data sinks) visible to the attacker, for which existential quantification over variables free in $e_{p}$ or $e_{l}$ is not necessary. A generalization to all precise predicates should be possible.

\subsection{Entailments}

Although implications between spatial formulas is not part of the assertion language, entailments $P \stackrel{\ell}{\Longrightarrow} Q$ between assertions still play a role in SECCSL's Hoare style consequence rule (CONSEQ in Fig. 4). We discuss entailment now as it sheds useful light on some consequences of SECCSL's relational semantics.

Definition 1 (Secure Entailment). $P \stackrel{\ell}{\Longrightarrow} Q$ holds iff

- $(s, h),\left(s^{\prime}, h^{\prime}\right) \models_{\ell} P$ implies $(s, h),\left.\left(s^{\prime}, h^{\prime}\right)\right|_{\ell} Q$ for all $s, h$ and $s^{\prime}, h^{\prime}$, and - $\operatorname{lows}_{\ell}(P, s) \subseteq \operatorname{lows}_{\ell}(Q, s)$ for all $s$

The security level $\ell$ is used not just in the evaluation of the assertions but also to preserve the $\ell$-attacker visible locations of $P$ in $Q$. This reflects the intuition that $P$ is stronger than $Q$, and so $Q$ should make fewer assumptions than $P$ on the limitations of an attacker's observational powers. 


\section{Proposition 1.}

$$
\begin{gathered}
e=e^{\prime} \wedge e_{l}=e_{l}{ }^{\prime} \wedge e:: e_{l} \stackrel{\ell}{\Longrightarrow} e^{\prime}:: e_{l}{ }^{\prime} \\
e:: e_{l} \wedge e_{l} \sqsubseteq e_{l}{ }^{\prime} \wedge e_{l}{ }^{\prime}:: \ell \stackrel{\ell}{\Longrightarrow} e:: e_{l}{ }^{\prime} \\
e_{l}:: \ell \stackrel{\ell}{\Longrightarrow} c:: e_{l} \quad \text { for a constant } c \\
e_{1}:: e_{l} \wedge \cdots \wedge e_{n}:: e_{l} \stackrel{\ell}{\Longrightarrow} f\left(e_{1}, \ldots, e_{n}\right):: e_{l} \quad \text { for } n>0 \\
e_{p} \stackrel{e_{l}}{\longrightarrow} e_{v} \wedge e_{l} \sqsubseteq \ell \stackrel{\ell}{\Longrightarrow} e_{p} \stackrel{e_{l}}{\longrightarrow} e_{v} \wedge e_{p}:: e_{l} \wedge e_{v}:: e_{l} \\
\left(\forall s . \operatorname{lows}_{\ell}(P, s)=\operatorname{lows}_{\ell}(Q, s)\right) \text { implies } \phi \wedge(\phi ? P: Q) \stackrel{\ell}{\Longrightarrow} P \\
P \stackrel{\ell}{\Longrightarrow} P^{\prime} \text { and } Q \stackrel{\ell}{\Longrightarrow} Q^{\prime} \text { implies } P \star Q \stackrel{\ell}{\Longrightarrow} P^{\prime} \star Q^{\prime}
\end{gathered}
$$

Entailment (14) in Proposition 1 shows that sensitivity of values is compatible with equality. This property fails in the security separation logic of [14], where labels are part of the semantics of expressions but are not compared by equality. The second property (15) captures the intuition that less-sensitive data can always be used in contexts where more-sensitive data might be expected (but not vice-versa). Recall that $e_{l}{ }^{\prime}$ here is an expression. The additional condition $e_{l}{ }^{\prime}:: \ell$ guarantees that this expression denotes a meaningful security level, i.e. evaluates identically in both states (cf. (7)). (abusing notation to let the semantic $\ell$ stand for some expression that denotes it). Property (16) encodes that constants do not depend on any state; again the security level expression $e_{l}$ must be meaningful, but trivially $c:: \ell$ when $\ell$ is constant, too. Value sensitivity is congruent with function application (17). This is not surprising, as functions map arguments equal in both states to equal results. Yet, as with (14) above, this property fails in [14] where security labels are attached to values. Note that the reverse entailment is false (e.g. for the constant function $\lambda x . c$ ).

Via (18), when $e_{p} \stackrel{e_{l}}{\longmapsto} e_{v}$ it follows that both the location $e_{p}$ and the value $e_{v}$ adhere to the level $e_{l}$, cf. (9). Note that the antecedent $e_{p} \stackrel{e_{l}}{\longmapsto} e_{v}$ is repeated in the consequent to ensure that the set of $\ell$-attacker visible locations is preserved. Conditional assertions can be resolved when the test is definite, provided that $P$ and $Q$ describe the same set of public locations, (19) and symmetrically for $\neg \phi$. Finally, separating conjunction is monotone wrt. entailment (20).

\subsection{Proof System}

We consider a canonical concurrent programming language with shared heap locations protected by locks but without shared variables. Commands $c$ comprise assignments to local variables, heap access (load and store), ${ }^{1}$ sequential programming constructs, as well as parallel composition and locking. We assume

\footnotetext{
${ }^{1}$ Volatile memory locations can be treated analogously to locks by introducing an additional assertion characterizing that part of the heap, that is implicitly available to atomic commands. This feature is realized in the Isabelle theories [18] but omitted here in the interests of brevity.
} 
a static collection of valid lock identifiers $l$, each of which has an assertion as its associated invariant $\operatorname{inv}(l)$, characterizing the protected portion of the heap. We describe the program semantics in Sect. 4 as part of the soundness proof.

$$
\begin{aligned}
c::= & x:=e\left|x:=\left[e_{v}\right]\right|\left[e_{p}\right]:=e_{v} \mid \text { lock } l \mid \text { unlock } l \\
& c_{1} ; c_{2}\left|c_{1} \| c_{2}\right| \text { if } b \text { then } c_{1} \text { else } c_{2} \mid \text { while } b \text { do } c
\end{aligned}
$$

The SECCSL proof rules are shown in Fig. 4. They extend the standard rules of concurrent separation logic [38] (CSL) by additional side-conditions that amount to information flow checks $e::$ _ as part of the respective preconditions.

Similarly to [46], without loss of generality we require that assignments (rules AsG, LOAD) are always to distinct variables, to avoid renaming in the assertions. In the postcondition of LOAD, $x:: e_{l}$ can be derived by CONSEQ for (18). Storing to a heap location through an $e_{l}$-sensitive location $e_{p} \stackrel{e_{l}}{\longmapsto} e_{v}$ (rule STORE) requires that the value $e_{v}$ written to that location admits the corresponding security level $e_{l}$ of the location $e_{p}$. Note that due to monotonicity (15) the security level does not have to match exactly. The rules for locking are standard [12]. To preclude information leaks through timing channels, the execution can branch on non-secret values only. This manifests in side conditions $b:: \ell$ for the respective branching condition $b$ where, recall, $\ell$ is the attacker security level (IF, WHILE). Logical SPLIT picks those two cases where $\llbracket \phi \rrbracket_{s}=\llbracket \phi \rrbracket_{s^{\prime}}$, ruling out the other two by $\phi:: \ell$. The consequence rule (CONSEQ) uses entailment relative to $\ell$ (Definition 1). Rule PAR has the usual proviso that the variables modified in one thread cannot interfere with those relied on by the other and its pre-/postcondition.

\section{Security Definition and Soundness}

The soundness theorem for SECCSL guarantees that if some triple $\ell \vdash\{P\} c\{Q\}$ is derived using the rules of Fig. 4, then: all executions of $c$ started in a state satisfying precondition $P$ are memory safe, partially correct with respect to postcondition $Q$, and moreover secure with respect to the sensitivity of values as denoted by $P$ and $Q$ and at all times respect the sensitivity of locations as denoted by $P$ (see Sect. 2.3). Proof outlines are relegated to Appendix B. All results have been mechanised in Isabelle/HOL [37] and are available at [18].

The top-level security property of SECCSL is a noninterference condition [19]. Noninterference as a security property specifies, roughly, that for any pair of executions that start in states that agree on the values of all attackerobservable inputs, then, from the attacker's point of view the resulting executions will be indistinguishable, i.e. all of the attacker visible observations will agree. In SECCSL, what is "attacker-observable" depends on the attacker level $\ell$. The "inputs" are the expressions $e$, and the attacker-visible inputs are those expressions $e$ whose sensitivity is given by $e:: \ell^{\prime}$ judgements in the precondition $P$ for which $\ell^{\prime} \sqsubseteq \ell$. The attacker-visible observations are the contents of all memory locations in $\operatorname{lows}_{\ell}(P, s)$, for initial store $s$ and precondition $P$. Thus we define when two heaps are indistinguishable to the $\ell$-attacker. 


$$
\begin{aligned}
& \frac{x \notin \operatorname{free}(e)}{\ell \vdash\{\operatorname{emp}\} x:=e\{x=e\}} \text { AsG } \quad \frac{x \notin \text { free }\left(e_{p}, e_{v}, e_{l}\right)}{\ell \vdash\left\{e_{p} \stackrel{e_{l}}{\longmapsto} e_{v}\right\} x:=\left[e_{p}\right]\left\{x=e_{v} \wedge e_{p} \stackrel{e_{l}}{\longmapsto} e_{v}\right\}} \text { LOAD } \\
& \overline{\ell \vdash\left\{e_{v}:: e_{l} \wedge e_{p} \stackrel{e_{l}}{\longmapsto} \_\right\}\left[e_{p}\right]:=e_{v}\left\{e_{p} \stackrel{e_{l}}{\longmapsto} e_{v}\right\}} \text { STORE } \\
& \overline{\ell \vdash\{\text { emp }\} \text { lock } l\{\operatorname{inv}(l)\}} \text { LOCK } \overline{\ell \vdash\{\operatorname{inv}(l)\} \text { unlock } l\{\text { emp }\}} \text { UNLOCK } \\
& \begin{array}{cc}
\ell \vdash\{b \wedge P\} c\{Q\} & \ell \vdash\{\phi \wedge P\} c\{Q\} \\
\ell \vdash\{\neg b \wedge P\} c\{Q\} & \ell \vdash\{\neg \phi \wedge P\} c\{Q\} \\
\frac{\ell \vdash\{b:: \ell \wedge P\} \text { if } b \text { then } c_{1} \text { else } c_{2}\{Q\}}{\ell} & \text { IF }
\end{array} \\
& \frac{\ell \vdash\{b \wedge b:: \ell \wedge P\} c\{b:: \ell \wedge P\}}{\ell \vdash\{b:: \ell \wedge P\} \text { while } b \text { do } c\{\neg b \wedge P\}} \text { WhiLE } \\
& \frac{\ell \vdash\{P\} c_{1}\{R\} \quad \ell \vdash\{R\} c_{2}\{Q\}}{\ell \vdash\{P\} c_{1} ; c_{2}\{Q\}} \text { SEQ } \quad \frac{\ell \vdash\{P\} c\{Q\}}{\ell \vdash\{P \star F\} c\{Q \star F\}} \text { Frame } \\
& P \stackrel{\ell}{\Longrightarrow} P^{\prime} \\
& Q^{\prime} \stackrel{\ell}{\Longrightarrow} Q \quad \operatorname{modified}\left(c_{i}\right) \cap \operatorname{free}\left(c_{j}, P_{j}, Q_{j}\right)=\varnothing \text { for } i \neq j \\
& \frac{\ell \vdash\left\{P^{\prime}\right\} c\left\{Q^{\prime}\right\}}{\ell \vdash\{P\} c\{Q\}} \text { ConseQ } \quad \frac{\ell \vdash\left\{P_{1}\right\} c_{1}\left\{P_{1}\right\} \quad \ell \vdash\left\{P_{2}\right\} c_{2}\left\{P_{2}\right\}}{\ell \vdash\left\{P_{1} \star P_{2}\right\} c_{1} \| c_{2}\left\{Q_{1} \star Q_{2}\right\}} \text { PAR }
\end{aligned}
$$

Fig. 4. Proof rules of SECCSL.

Definition 2 ( $\ell$ Equivalence). Two heaps coincide on a set of locations $A$, written $h \equiv_{A} h^{\prime}$, iff for all $a \in A$. $a \in \operatorname{dom}(h) \cap \operatorname{dom}\left(h^{\prime}\right)$ and $h(a)=h^{\prime}(a)$. Two heaps $h$ and $h^{\prime}$ are $\ell$-equivalent wrt. store $s$ and assertion $P$, if $h \equiv_{A} h^{\prime}$ for $A=\operatorname{lows}_{\ell}(P, s)$.

Then, the $\ell$-validity of an assertion $P$ in the relational semantics witnesses $\ell$ equivalence between the corresponding heaps.

Lemma 1. If $(s, h),\left(s^{\prime}, h^{\prime}\right) \models_{\ell} P$, then $h \equiv_{A} h^{\prime}$ for $A=\operatorname{lows}_{\ell}(P, s)$.

Furthermore, if $(s, h),\left(s^{\prime}, h^{\prime}\right) \models_{\ell} P$, then $\operatorname{lows}_{\ell}(P, s)=\operatorname{lows}_{\ell}\left(P, s^{\prime}\right)$ since the security levels in labeled points-to predicates must coincide on $s$ and $s^{\prime}$, cf. (9).

Semantics. Semantic configurations, denoted by $k$ in the following, are one of three kinds: (run $c, L, s, h$ ) denotes a command $c$ in a state $s, h$ where $L$ is a set of locks that are currently not held by any thread and can be acquired by $c$; ( $\operatorname{stop} L, s, h$ ) similarly denotes a final state $s, h$ with residual locks $L$, and abort results from invalid heap access.

The single-step relation (run $c, L, s, h) \stackrel{\sigma}{\longrightarrow} k$ takes running configurations to successors $k$ with respect to a schedule $\sigma$ that resolves the non-determinism of parallel composition. The schedule $\sigma$ is a list of actions: the action $\langle\tau\rangle$ represents the execution of atomic commands and the evaluation of conditionals; 
the actions $\langle 1\rangle$ and $\langle 2\rangle$ respectively denote the execution of the left- and righthand sides of a parallel composition for a single step, and so define a deterministic scheduling discipline reminiscent of separation kernels [32]. For example, $\left(\operatorname{run} c_{1} \| c_{2}, L, s, h\right) \stackrel{\langle 1\rangle \cdot \sigma}{\longrightarrow}\left(\operatorname{run} c_{1}^{\prime} \| c_{2}, L^{\prime}, s^{\prime}, h^{\prime}\right)$ if $\left(\operatorname{run} c_{1}, L, s, h\right) \stackrel{\sigma}{\longrightarrow}$ (run $c_{1}^{\prime}, L^{\prime}, s^{\prime}, h^{\prime}$ ). Configurations (run lock $l, L, s, h$ ) can only be scheduled if $l \in L$ (symmetrically for unlock)) and otherwise block without a possible step.

Executions $k_{1} \stackrel{\sigma_{1} \cdots \sigma_{n}}{\longrightarrow} k_{n+1}$ chain several steps $k_{i} \stackrel{\sigma_{i}}{\longrightarrow} k_{i+1}$ by accumulating the schedule. We are considering partial correctness only, thus the schedule is always finite and so are all executions. The rules for program steps are otherwise standard and can be found in Appendix A.

Compositional Security. To prove that SECCSL establishes its top-level noninterference condition, we first define a compositional security condition that provides the central characterization of security for a command $c$ with respect to precondition $P$ and postcondition $Q$. That central, compositional property we denote $\operatorname{secure}_{\ell}^{n}(P, c, Q)$ and formalize below in Definition 3. It ensures that the first $n$ steps (or fewer if the program terminates before that) are safe and preserve $\ell$-equivalence of the heap locations specified initially in $P$, but in a way that is compositional across multiple execution steps, across multiple threads of execution and across different parts of the heap. It is somewhat akin, although more precise than, prior characterizations based on strong low bisimulation [16,45].

Disregarding the case when $c$ terminates before the $n$-th step for a moment, for a pair of initial states $\left(s_{1}, h_{1}\right)$ and $\left(s_{1}^{\prime}, h_{1}^{\prime}\right)$ and initial set of locks $L_{1}$, and a fixed schedule $\sigma=\sigma_{1} \cdots \sigma_{n}$, secure $_{\ell}^{n+1}\left(P_{1}, c_{1}, Q\right)$ requires that $c$ performs a sequence of lockstep execution steps from each initial state

$$
\begin{aligned}
& \left(\operatorname{run} c_{i}, L_{i}, s_{i}, h_{i}\right) \stackrel{\sigma_{i}}{\longrightarrow}\left(\operatorname{run} c_{i+1}, L_{i+1}, s_{i+1}, h_{i+1}\right) \quad \text { for } 1 \leq i \leq n \\
& \left(\operatorname{run} c_{i}, L_{i}, s_{i}^{\prime}, h_{i}^{\prime}\right) \stackrel{\sigma_{i}}{\longrightarrow}\left(\operatorname{run} c_{i+1}, L_{i+1}, s_{i+1}^{\prime}, h_{i+1}^{\prime}\right)
\end{aligned}
$$

These executions must agree on the intermediate commands $c_{i}$ and locks $L_{i}$ and the $i$ th pair of states must satisfy an intermediate assertion of the following form:

$$
\left(s_{i}, h_{i}\right),\left(s_{i}^{\prime}, h_{i}^{\prime}\right) \models_{\ell} P_{i} \star F \star \operatorname{invs}\left(L_{i}\right) \quad \text { where } \operatorname{invs}\left(L_{i}\right)=\star_{l_{i} \in L_{i}} \operatorname{inv}\left(l_{i}\right)
$$

Here $P_{i}$ describes the part of the heap that command $c_{i}$ is currently accessing. $\operatorname{invs}\left(L_{i}\right)$ is the set of lock invariants for the locks $l_{i} \in L_{i}$ not currently acquired. Its presence ensures that whenever a lock is acquired that the associated invariant can be assumed to hold. Finally $F$ is an arbitrary frame, an assertion that does not mention variables updated by $c_{i}$. Its inclusion allows the security property to compose with respect to different parts of the heap.

Moreover, each $P_{i+1} \star \operatorname{invs}\left(L_{i+1}\right)$ is required to preserve the sensitivity of all $\ell$-visible heap locations of $P_{i} \star \operatorname{invs}\left(L_{i}\right)$, i.e. so that $\operatorname{lows}_{\ell}\left(P_{i} \star \operatorname{invs}\left(L_{i}\right), s_{i}\right) \subseteq$ $\operatorname{lows}_{\ell}\left(P_{i+1} \star \operatorname{invs}\left(L_{i+1}\right), s_{i+1}\right)$. If some intermediate step $m \leq n$ terminates, then $P_{m+1}=Q$, ensuring the postcondition holds when the executions terminate. Lastly, neither execution is allowed to reach an abort configuration. 
If the initial state satisfies $P_{1} \star F \star \operatorname{invs}\left(L_{1}\right)$ then $(22)$ holds throughout the entire execution, and establishes the end-to-end property that any final state indeed satisfies the postcondition and that $\operatorname{lows}_{\ell}\left(P_{1} \star \operatorname{invs}\left(L_{1}\right), s_{1}\right) \subseteq \operatorname{lows}_{\ell}\left(P_{i} \star\right.$ $\left.\operatorname{invs}\left(L_{i}\right), s_{i}\right)$ with respect to the initially specified low locations.

The property $\operatorname{secure}_{\ell}^{n}(P, c, Q)$ is defined recursively to match the steps of the lockstep execution of the program.

\section{Definition 3 (Security).}

- $\operatorname{secure}_{\ell}^{0}\left(P_{1}, c_{1}, Q\right)$ holds always.

- secure $_{\ell}^{n+1}\left(P_{1}, c_{1}, Q\right)$ holds, iff for all pairs of states $\left(s_{1}, h_{1}\right),\left(s_{1}^{\prime}, h_{1}^{\prime}\right)$, frames $F$, and sets of locks $L_{1}$, such that $\left(s_{1}, h_{1}\right),\left(s_{1}^{\prime}, h_{1}^{\prime}\right)=\ell P_{1}$ * $F \star \operatorname{invs}\left(L_{1}\right)$, and given two steps (run $\left.c_{1}, L_{1}, s_{1}, h_{1}\right) \stackrel{\sigma}{\longrightarrow} k$ and (run $\left.c_{1}, L_{1}, s_{1}^{\prime}, h_{1}^{\prime}\right) \stackrel{\sigma}{\longrightarrow} k^{\prime}$ there exists an assertion $P_{2}$ and a pair of successor states with either of

- $k=\left(\operatorname{stop} L_{2}, s_{2}, h_{2}\right)$ and $k^{\prime}=\left(\operatorname{stop} L_{2}, s_{2}^{\prime}, h_{2}^{\prime}\right)$ and $P_{2}=Q$

- $k=\left(\right.$ run $\left.c_{2}, L_{2}, s_{2}, h_{2}\right)$ and $k^{\prime}=\left(\right.$ run $\left.c_{2}, L_{2}, s_{2}^{\prime}, h_{2}^{\prime}\right)$ with $\operatorname{secure}_{\ell}^{n}\left(P_{2}, c_{2}, Q\right)$

such that $\left(s_{2}, h_{2}\right),\left(s_{2}^{\prime}, h_{2}^{\prime}\right) \models_{\ell} P_{2} \star F \star \operatorname{invs}\left(L_{2}\right)$ and $\operatorname{lows}_{\ell}\left(P_{1} \star\right.$ $\left.\operatorname{invs}\left(L_{1}\right), s_{1}\right) \subseteq \operatorname{lows}_{\ell}\left(P_{2} \star \operatorname{invs}\left(L_{2}\right), s_{2}\right)$ in both cases.

Two further side condition are imposed, ensuring all mutable shared state lies in the heap (cf. Sect. 3): $c_{1}$ doesn't modify variables occurring in $\operatorname{invs}\left(L_{1}\right)$ and $F$ (which guarantees that both remain intact), and the free variables in $P_{2}$ can only mention those already present in $P_{1}, c_{1}$, or in any lock invariant (which guarantees that $P_{2}$ remains stable against concurrent assignments). Note that each step can pick a different frame $F$, as required for the soundness of rule PAR.

Lemma 2. $\ell \vdash\{P\} c\{Q\}$ implies $\operatorname{secure}_{\ell}^{n}(P, c, Q)$ for every $n \geq 0$.

Safety, Correctness and Noninterference. Execution safety and correctness with respect to pre- and postcondition follow straightforwardly from Lemma 2.

Corollary 1 (Safety). Given initial states $\left(s_{1}, h_{1}\right),\left(s_{1}^{\prime}, h_{1}^{\prime}\right) \models_{\ell} P \star \operatorname{invs}\left(L_{1}\right)$ and two executions of a command $c$ under the same schedule to resulting configurations $k$ and $k^{\prime}$ respectively, then $\ell \vdash\{P\} c\{Q\}$ implies $k \neq$ abort $\wedge k^{\prime} \neq$ abort.

Theorem 1 (Correctness). For initial states $\left(s_{1}, h_{1}\right),\left(s_{1}^{\prime}, h_{1}^{\prime}\right) \models_{\ell} P$ * $\operatorname{invs}\left(L_{1}\right)$, given two complete executions of a command $c$ under the same schedule $\sigma$

$$
\begin{aligned}
& \left(\operatorname{run} c, L_{1}, s_{1}, h_{1}\right) \stackrel{\sigma}{\longrightarrow} *\left(\operatorname{stop} L_{2}, s_{2}, h_{2}\right) \\
& \left(\operatorname{run} c_{i}, L_{i}, s_{i}^{\prime}, h_{i}^{\prime}\right) \stackrel{\sigma}{\longrightarrow} *\left(\operatorname{stop} L_{2}, s_{2}^{\prime}, h_{2}^{\prime}\right)
\end{aligned}
$$

then $\ell \vdash\{P\} c\{Q\}$ implies $\left(s_{2}, h_{2}\right),\left(s_{2}^{\prime}, h_{2}^{\prime}\right) \models_{\ell} Q \star \operatorname{invs}\left(L_{2}\right)$.

The top-level noninterference property also follows from Lemma 2 via Lemma 1. For brevity, we state the noninterference property directly in the theorem: 
Theorem 2 (Noninterference). Given a command $c$, and initial states $\left(s_{1}, h_{1}\right),\left(s_{1}^{\prime}, h_{1}^{\prime}\right) \models_{\ell} P \star \operatorname{invs}\left(L_{1}\right)$ then $\ell \vdash\{P\} c\{Q\}$ implies $h_{i} \equiv_{A} h_{i}^{\prime}$, where $A=\operatorname{lows}_{\ell}\left(P \star \operatorname{invs}\left(L_{1}\right), s_{1}\right)$, for all pairs of heaps $h_{i}$ and $h_{i}^{\prime}$ arising from executing the same schedule from each initial state.

\section{SECC: Automating SECCSL}

To demonstrate the ease by which SECCSL can be automated, we develop the prototype tool SECC, available at [18]. It implements the logic from Sect. 3 for a subset of C. SECC is currently used to explore reasoning about example programs with interesting security policies. Thus its engineering has focused on features related to security reasoning (e.g. deciding when conditions $e:: e_{l}$ are entailed) rather than reasoning about complex data structures.

Symbolic Execution. SECC automates SECCSL through symbolic execution, as pioneered for SL in [7]. Similarly to VeriFast's algorithm in [22], the verifier computes the strongest postcondition of a command $c$ when executed in a symbolic state, yielding a set of possible final symbolic states. Each such state $\sigma=(\rho, s, \underline{P})$ maintains a path condition $\rho$ of relational formulas (from procedure contracts, invariants, and the evaluation of conditionals) and a symbolic heap described by a list $\underline{P}=\left(P_{1} \star \cdots \star P_{n}\right)$ of atomic spatial assertions (points-to and instances of defined predicates). The symbolic store $s$ maps program variables to pure expressions, where $s(e)$ denotes substituting $s$ into $e$. As an example, when $P_{i}=s\left(e_{p}\right) \mapsto v$ is part of the symbolic heap, a load $x:=e_{p}$ in $\sigma$ can be executed to yield the updated state $(\rho, s(x:=v), \underline{P})$ where $x$ is mapped to $v$.

To find the $P_{i}$ we match the left-hand sides of points-to predicates. Similarly, matching is used during checking of entailments $\rho_{1} \wedge \underline{P} \stackrel{\ell}{\Longrightarrow} \exists \underline{x} \cdot \rho_{2} \wedge \underline{Q}$, where the conclusion is normalized to prenex form. The entailment is reduced to a non-spatial problem by incrementally computing a substitution $\tau$ for the existentials $\underline{x}$, removing pairs $P_{i}=\tau\left(Q_{j}\right)$ in the process, as justified by (20) (see also "subtraction rules" in [7, Sec. 4]).

Finally, the remaining relational problem $\rho_{1} \Rightarrow \rho_{2}$ without spatial connectives can be encoded into first-order [17], by duplicating the pure formulas in terms of fresh variables to represent the second state, and by the syntactic equivalent of (7). The resulting verification condition is discharged with Z3 [15]. This translation is semantically complete. For example, consider Fig. 4 from Prabawa et al. [43]. It has a conditional if $(\mathrm{b}==\mathrm{b}) \ldots$, whose check $(b=b)::$ low, translated to $(b=b)=\left(b^{\prime}=b^{\prime}\right)$ by SECC, holds independently of $b^{\prime}$ 's sensitivity.

Features. In addition to the logic from Sect. 3, SECC supports procedure modular verification with pre-/postconditions as usual; and it supports user-defined spatial predicates. While some issues of the $\mathrm{C}$ source language are not addressed (yet), such as integer overflow, those that impact directly on information flow security are taken into account. Specifically, the shortcut semantics of boolean operators $\& \&,||$, and ternary _ $?_{-}:_{-}$count as branching points and as such 
the left hand side resp. the test must not depend on sensitive data, similarly to the conditions of if statements and while loops.

A direct benefit of the integration of security levels into the assertion language is that it becomes possible to specify the sensitivity of data passed to library and operating system functions. For example, the execution time of malloc(len) would depend on the value of len, which can thus be required to satisfy len :: low by annotating its function header with an appropriate precondition, using SECC's requires annotation. Likewise, SECC can reason about limited forms of declassification, in which external functions are trusted to safely release otherwise sensitive data, by giving them appropriate pre-/postconditions. For example, a password hashing library function prototype might be annotated with a postcondition asserting its result is low, via SECC's ensures annotation.

Examples and Case Study. SECC proves Fig. 1 secure, and correctly flags buggy variants as insecure, e.g., where the test in thread 1 is reversed, or when thread 2 does not clear the data field upon setting the is_classified to FALSE. SECC also correctly analyzes those 7 examples from [17] that are supported by the logic and tool (each in $\sim 10 \mathrm{~ms}$ ). All examples are available at [18].

To compare SECC and SECCSL against the recent Covern logic [34], we took a non-trivial example program that Murray et al. verified in CovERN, manually translated it to C, and verified it automatically using SECC. The original program $^{2}$, written in CoverN's tiny While language embedded in Isabelle/HOL, models the software functionality of a simplified implementation of the Cross Domain Desktop Compositor (CDDC) [5]. The CDDC is a device that facilitates interactions with multiple PCs, each of which runs applications at differing sensitivity, from a single keyboard, mouse and display. Its multi-threaded software handles routing of keyboard input to the appropriate PC and switching between the PCs via mouse gestures. Verifying the $\mathrm{C}$ translation required adding SECCSL annotations for procedure pre-/postconditions and loop invariants. The $\mathrm{C}$ translation including those annotations is $\sim 250$ lines in length. The present, unoptimised, implementation of SECC verifies the resulting artifact in $\sim 5 \mathrm{~s}$. In contrast, the COVERN proof of this example requires $\sim 600$ lines of Isabelle/HOL definitions/specification, plus $\sim 550$ lines of Isabelle proof script.

\section{Related Work}

There has been much work targeting type systems and program logics for concurrent information flow. Karbyshev et al. [23] provide an excellent overview. Here we concentrate on work whose ideas are most closely related to SECCSL.

Costanzo and Shao [14] propose a sequential separation logic for reasoning about information flow. Unlike SECCSL, theirs does not distinguish value and location sensitivity. Their separation logic assertions have a fairly standard (nonrelational) semantics, at the price of having a security-aware language semantics

\footnotetext{
${ }^{2}$ https://bitbucket.org/covern/covern/src/master/examples/cddc/Example_CDDC_ WhileLockLanguage.thy.
} 
that propagates security labels attached to values in the store and heap. As mentioned in Sect. 3.2, this has the unfortunate side-effect of breaking intuitive properties about sensitivity assertions. We conjecture that the absence of such properties would make their logic harder to automate than SECCSL, which SECC demonstrates is feasible. SECCSL avoids the aforementioned drawbacks by adopting a relational assertion semantics.

Gruetter and Murray [20] propose a security separation logic in Coq [8] for Verifiable C, the C subset of the Verified Software Toolchain [2,3]. However they provide no soundness proof for its rules and its feasibility to automate is unclear.

Two recent compositional logics for concurrent information flow are the CovERN logic [34] and the type and effect system of Karbyshev et al. [23]. Both borrow ideas from separation logic. However, unlike SECCSL, neither is defined for languages with pointers, arrays etc.

Like SECCSL, COVERn proves a timing-sensitive security property. Location sensitivity is defined statically by value-dependent predicates, and value sensitivity is tracked by a dependent security typing context $\Gamma$ [35], relative to a Hoare logic predicate $P$ over the entire shared memory. In Covern locks carry non-relational invariants. In contrast, SECCSL unifies these elements together into separation logic assertions with a relational semantics. Doing so leads to a much simpler logic, amenable to automation, while supporting pointers, etc.

On the other hand, Karbyshev et al. [23] prove a timing-insensitive security property, but rely on primitives to interact with the scheduler to prevent leaks via scheduling decisions. Unlike SECCSL, which assumes a deterministic scheduling discipline, Karbyshev et al. support a wider class of scheduling policies. Their system tracks resource ownership and transfer between threads at synchronisation points, similar to CSLs. Their resources include labelled scheduler resources that account for scheduler interaction, including when scheduling decisions become tainted by secret data - something that cannot occur in SECCSL's deterministic scheduling model.

Prior logics for sequential languages, e.g. [1,4], have also adopted separation logic ideas to reason locally about memory, combining them with relational assertions similar to SECCSL's $e:: e_{l}$ assertions. For instance, the agreement assertions A $(e)$ of [4] coincide with SECCSL's $e$ :: low. Unlike SECCSL, some of these logics support languages with explicit declassification actions [4].

Self-composition is another technique to exploit existing verification infrastructure for proofs of general hyperproperties [13], including but not limited to non-interference. Eilers et al. [17] present such an approach for Viper, which supports an assertion language similar to that of separation logic. It does not support public heap locations (which are information sources and sinks at the same time) albeit sinks can be modeled via preconditions of procedures. A similar approach is implemented in Frama-C [9]. Both of $[9,17]$ do not support concurrency, and it remains unclear how self-composition could avoid an exponential blow-up from concurrent interleaving, which SECCSL avoids.

The soundness proof for SECCSL follows the general structure of Vafeiadis' [46] for CSL, which is also mechanised in Isabelle/HOL. There is, 
however, a technical difference: His analog of Definition 3, a recursive predicate called $\operatorname{safe}_{n}(c, s, h, Q)$, refers to a semantic initial state $s, h$ whereas we propagate a syntactic assertion (22) only. Our formulation has the benefit that some of the technical reasoning in the soundness proof is easier to automate. Its drawback is the need to impose technical side-conditions on the free variables of the frame $F$ and the intermediate assertions $P_{i}$.

\section{Conclusion}

We presented SECCSL, a concurrent separation logic for proving expressive datadependent information flow properties of programs. SECCSL is considerably simpler, yet handles features like pointers, arrays etc., which are out of scope for contemporary logics. It inherits the structure of traditional concurrent separation logics, and so like those logics can be automated via symbolic execution [10, $22,30]$. To demonstrate this, we implemented SECC, an automatic verifier for expressive information flow security for a subset of the $\mathrm{C}$ language.

Separation logic has proved to be a remarkably powerful vehicle for reasoning about programs, weak memory concurrency [47], program synthesis [42], and many other domains. With SECCSL, we hope that in future the same possibilities might be opened to verified information flow security.

Acknowledgement. We thank the anonymous reviewers for their careful and detailed comments that helped significantly to clarify the discussion of finer points.

This research was sponsored by the Department of the Navy, Office of Naval Research, under award \#N62909-18-1-2049. Any opinions, findings, and conclusions or recommendations expressed in this material are those of the author(s) and do not necessarily reflect the views of the Office of Naval Research.

\section{A Command Semantics}

Symmetric parallel rules in which $c_{2}$ is scheduled under the action $\langle 2\rangle$ omitted.

$$
\begin{aligned}
& \frac{s^{\prime}=s\left(x \mapsto \llbracket e \rrbracket_{s}\right)}{(\operatorname{run} x:=e, L, s, h) \stackrel{\langle\tau\rangle}{\longrightarrow}\left(\operatorname{stop} L, s^{\prime}, h\right)} \quad \frac{\llbracket e \rrbracket_{s} \notin \operatorname{dom}(h)}{(\operatorname{run} x:=[e], L, s, h) \stackrel{\langle\tau\rangle}{\longrightarrow} \text { abort }} \\
& \underline{\llbracket e \rrbracket_{s} \in \operatorname{dom}(h) \quad s^{\prime}=s\left(x \mapsto h\left(\llbracket e \rrbracket_{s}\right)\right)} \\
& (\operatorname{run} x:=[e], L, s, h) \stackrel{\langle\tau\rangle}{\longrightarrow}\left(\operatorname{stop} L, s^{\prime}, h\right) \\
& \llbracket e_{1} \rrbracket_{s} \notin \operatorname{dom}(h) \\
& \text { (run } \left.\left[e_{1}\right]:=e_{2}, L, s, h\right) \stackrel{\langle\tau\rangle}{\longrightarrow} \text { abort } \\
& \underline{\llbracket e_{1} \rrbracket_{s} \in \operatorname{dom}(h) \quad h^{\prime}=h\left(\llbracket e_{1} \rrbracket_{s} \mapsto \llbracket e_{2} \rrbracket_{s}\right)} \\
& \left(\operatorname{run}\left[e_{1}\right]:=e_{2}, L, s, h\right) \stackrel{\langle\tau\rangle}{\longrightarrow}\left(\operatorname{stop} L, s, h^{\prime}\right) \\
& l \in L \quad L^{\prime}=L \backslash\{l\} \\
& \overline{\text { (run lock } l, L, s, h) \stackrel{\langle\tau\rangle}{\longrightarrow}\left(\operatorname{stop} L^{\prime}, s, h\right)}
\end{aligned}
$$




$$
\begin{aligned}
& \frac{l \notin L \quad L^{\prime}=L \cup\{l\}}{(\text { run unlock } l, L, s, h) \stackrel{\langle\tau\rangle}{\longrightarrow}\left(\operatorname{stop} L^{\prime}, s, h\right)} \quad \frac{\left(\operatorname{run} c_{1}, L, s, h\right) \stackrel{\sigma}{\longrightarrow} \text { abort }}{\left(\operatorname{run} c_{1} ; c_{2}, L, s, h\right) \stackrel{\sigma}{\longrightarrow} \text { abort }} \\
& \frac{\left(\operatorname{run} c_{1}, L, s, h\right) \stackrel{\sigma}{\longrightarrow} \text { abort }}{\left(\operatorname{run} c_{1} \| c_{2}, L, s, h\right) \stackrel{\langle 1\rangle \cdot \sigma}{\longrightarrow} \text { abort }} \quad \frac{\left(\operatorname{run} c_{1}, L, s, h\right) \stackrel{\sigma}{\longrightarrow}\left(\operatorname{stop} L^{\prime}, s^{\prime}, h^{\prime}\right)}{\left(\operatorname{run} c_{1} ; c_{2}, L, s, h\right) \stackrel{\sigma}{\longrightarrow}\left(\operatorname{run} c_{2}, L^{\prime}, s^{\prime}, h^{\prime}\right)} \\
& \frac{\left(\operatorname{run} c_{1}, L, s, h\right) \stackrel{\sigma}{\longrightarrow}\left(\operatorname{run} c_{1}^{\prime}, L^{\prime}, s^{\prime}, h^{\prime}\right)}{\left(\operatorname{run} c_{1} ; c_{2}, L, s, h\right) \stackrel{\sigma}{\longrightarrow}\left(\operatorname{run} c_{1}^{\prime} ; c_{2}, L^{\prime}, s^{\prime}, h^{\prime}\right)} \\
& \frac{\left(\text { run } c_{1}, L, s, h\right) \stackrel{\sigma}{\longrightarrow}\left(\operatorname{stop} L^{\prime}, s^{\prime}, h^{\prime}\right)}{\left(\text { run } c_{1} \| c_{2}, L, s, h\right) \stackrel{\langle 1\rangle \cdot \sigma}{\longrightarrow}\left(\text { run } c_{2}, L^{\prime}, s^{\prime}, h^{\prime}\right)} \\
& \frac{\left(\operatorname{run} c_{1}, L, s, h\right) \stackrel{\sigma}{\longrightarrow}\left(\operatorname{run} c_{1}^{\prime}, L^{\prime}, s^{\prime}, h^{\prime}\right)}{\left(\operatorname{run} c_{1} \| c_{2}, L, s, h\right) \stackrel{\langle 1\rangle \cdot \sigma}{\longrightarrow}\left(\operatorname{run} c_{1}^{\prime} \| c_{2}, L^{\prime}, s^{\prime}, h^{\prime}\right)} \\
& \frac{\text { if } s \models b \text { then } c^{\prime}=c_{1} \text { else } c^{\prime}=c_{2}}{\text { (run if } \left.b \text { then } c_{1} \text { else } c_{2}, L, s, h\right) \stackrel{\langle\tau\rangle}{\longrightarrow}\left(\operatorname{run} c^{\prime}, L, s, h\right)} \\
& s \not \neq b \\
& \overline{\text { (run while } b \text { do } c, L, s, h) \stackrel{\langle\tau\rangle}{\longrightarrow}(\operatorname{stop} L, s, h)} \\
& s \models b \\
& \overline{(\text { run } \underbrace{\text { while } b \text { do } c}_{\omega}, L, s, h) \stackrel{\langle\tau\rangle}{\longrightarrow}(\operatorname{run}(c ; \omega), L, s, h)} \\
& k \stackrel{\langle\rangle}{\longrightarrow} k \quad \frac{k \stackrel{\sigma_{1}}{\longrightarrow} k^{\prime} \quad k^{\prime} \stackrel{\sigma_{2}}{\longrightarrow} * k^{\prime \prime}}{k \stackrel{\sigma_{1} \cdot \sigma_{2}}{\longrightarrow} * k^{\prime \prime}}
\end{aligned}
$$

\section{B Proofs}

\section{Proof of Lemma 1}

If $(s, h),\left(s^{\prime}, h^{\prime}\right) \models_{\ell} P$, then $h \stackrel{A}{\equiv} h^{\prime}$ for $A=\operatorname{lows}_{\ell}(P, s)$.

Proof. By induction on the structure of $P$, noting that $\operatorname{lows}_{\ell}\left({ }_{-}, s\right)$ contains locations of the corresponding sub-heap only. 


\section{Proof of Lemma 2}

$\ell \vdash\{P\} c\{Q\}$ implies $\operatorname{secure}_{\ell}^{n}(P, c, Q)$ for every $n \geq 0$.

Proof (Outline). By induction on the derivation of the validity of the judgement. Noting that $n=0$ is trivial, we may unfold the recursion of the security definition once to prove the base cases of assignment, load, store, and locking, which then follow from the respective side conditions of the proof rules.

For rules IF and While, the side condition $b:: \ell$ guarantees that the test evaluates equivalently in the two states and thus execution proceeds with the same remainder program.

Except for IF, all remaining rules need a second induction on $n$ to stepwise match security of the premise to security of the conclusion (e.g. over the steps of the first command in a sequential composition $c_{1} ; c_{2}$ ).

The rule Frame instantiates the frame $F$ with the same assertion in each step, whereas PAR uses the frame $F$ to preserve the current precondition $P_{2}$ of $c_{2}$ over steps of $c_{1}$ and vice-versa.

\section{Proof of Corollary 1}

Given a command $c$ and initial states $\left(s_{1}, h_{1}\right),\left(s_{1}^{\prime}, h_{1}^{\prime}\right) \models_{\ell} P \star \operatorname{invs}\left(L_{1}\right)$ and two executions under the same schedule to resulting configurations $k$ and $k^{\prime}$ respectively, then $\ell \vdash\{P\} c\{Q\}$ implies $k \neq$ abort $\wedge k^{\prime} \neq$ abort.

Proof. By induction on the number of steps $n$ of the executions from $\operatorname{secure}_{\ell}^{n}(P, c, Q)$ via Lemma 2 .

\section{Proof of Theorem 1}

Given a command $c$ and initial states $\left(s_{1}, h_{1}\right),\left(s_{1}^{\prime}, h_{1}^{\prime}\right) \models_{\ell} P \star \operatorname{invs}\left(L_{1}\right)$ and two complete executions under the same schedule $\sigma$

$$
\begin{aligned}
& \left(\text { run } c, L_{1}, s_{1}, h_{1}\right) \stackrel{\sigma}{\longrightarrow} *\left(\operatorname{stop} L_{2}, s_{2}, h_{2}\right) \\
& \left(\text { run } c_{i}, L_{i}, s_{i}^{\prime}, h_{i}^{\prime}\right) \stackrel{\sigma}{\longrightarrow}\left(\operatorname{stop} L_{2}, s_{2}^{\prime}, h_{2}^{\prime}\right)
\end{aligned}
$$

then $\ell \vdash\{P\} c\{Q\}$ implies $\left(s_{2}, h_{2}\right),\left(s_{2}^{\prime}, h_{2}^{\prime}\right) \models_{\ell} Q \star \operatorname{invs}\left(L_{2}\right)$.

Proof. By induction on the number of steps $n$ of the executions from $\operatorname{secure}_{\ell}^{n}(P, c, Q)$ via Lemma 2.

\section{Proof of Theorem 2}

Given a command $c$, and initial states $\left(s_{1}, h_{1}\right),\left(s_{1}^{\prime}, h_{1}^{\prime}\right) \models_{\ell} P \star \operatorname{invs}\left(L_{1}\right)$ then $\ell \vdash\{P\} c\{Q\}$ implies $h_{i} \stackrel{A}{=} h_{i}^{\prime}$, where $A=\operatorname{lows}_{\ell}\left(P, s_{1}\right)$, for all pairs of heaps $h_{i}$ and $h_{i}^{\prime}$ arising from executing the same schedule from each initial state.

Proof. By induction on the number of steps $i$ up to that state from $\operatorname{secure}_{\ell}^{i}(P, c, Q)$ via Lemma 2 we have $\operatorname{lows}_{\ell}\left(P \star \operatorname{invs}\left(L_{1}\right), s_{1}\right) \subseteq \operatorname{lows}_{\ell}\left(P_{i} \star\right.$ $\left.\operatorname{invs}\left(L_{1}\right), s_{i}\right)$ transitively over the prefix, where $P_{i}$ and $s_{i}$ are from the $i$-th state. The theorem then follows from Lemma 1 in Sect.3.1. 


\section{References}

1. Amtoft, T., Bandhakavi, S., Banerjee, A.: A logic for information flow in objectoriented programs. In: Proceedings of Principles of Programming Languages (POPL), pp. 91-102. ACM (2006)

2. Appel, A.W., et al.: Program Logics for Certified Compilers. Cambridge University Press, New York (2014)

3. Appel, A.W., et al.: The Verified Software Toolchain (2017). https://github.com/ PrincetonUniversity/VST

4. Banerjee, A., Naumann, D.A., Rosenberg, S.: Expressive declassification policies and modular static enforcement. In: Proceedings of Symposium on Security and Privacy (S\&P), pp. 339-353. IEEE (2008)

5. Beaumont, M., McCarthy, J., Murray, T.: The cross domain desktop compositor: using hardware-based video compositing for a multi-level secure user interface. In: Annual Computer Security Applications Conference (ACSAC), pp. 533-545. ACM (2016)

6. Benton, N.: Simple relational correctness proofs for static analyses and program transformations. In: Proceedings of Principles of Programming Languages (POPL), pp. 14-25. ACM (2004)

7. Berdine, J., Calcagno, C., O'Hearn, P.W.: Symbolic execution with separation logic. In: Yi, K. (ed.) APLAS 2005. LNCS, vol. 3780, pp. 52-68. Springer, Heidelberg (2005). https://doi.org/10.1007/11575467_5

8. Bertot, Y., Castéran, P.: Interactive Theorem Proving and Program Development. Coq'Art: The Calculus of Inductive Constructions. Texts in Theoretical Computer Science. An EATCS Series. Springer, Heidelberg (2004). https://doi.org/10.1007/ 978-3-662-07964-5

9. Blatter, L., Kosmatov, N., Le Gall, P., Prevosto, V., Petiot, G.: Static and dynamic verification of relational properties on self-composed C code. In: Dubois, C., Wolff, B. (eds.) TAP 2018. LNCS, vol. 10889, pp. 44-62. Springer, Cham (2018). https:// doi.org/10.1007/978-3-319-92994-1_3

10. Calcagno, C., Distefano, D.: Infer: an automatic program verifier for memory safety of C programs. In: Bobaru, M., Havelund, K., Holzmann, G.J., Joshi, R. (eds.) NFM 2011. LNCS, vol. 6617, pp. 459-465. Springer, Heidelberg (2011). https:// doi.org/10.1007/978-3-642-20398-5_33

11. Calcagno, C., et al.: Moving fast with software verification. In: Havelund, K., Holzmann, G., Joshi, R. (eds.) NFM 2015. LNCS, vol. 9058, pp. 3-11. Springer, Cham (2015). https://doi.org/10.1007/978-3-319-17524-9_1

12. Chlipala, A.: Formal Reasoning About Programs (2016)

13. Clarkson, M.R., Schneider, F.B.: Hyperproperties. In: Proceedings of Computer Security Foundations Symposium (CSF), pp. 51-65 (2008)

14. Costanzo, D., Shao, Z.: A separation logic for enforcing declarative information flow control policies. In: Abadi, M., Kremer, S. (eds.) POST 2014. LNCS, vol. 8414, pp. 179-198. Springer, Heidelberg (2014). https://doi.org/10.1007/978-3-642-547928 _ 10

15. de Moura, L., Bjørner, N.: Z3: an efficient SMT solver. In: Ramakrishnan, C.R., Rehof, J. (eds.) TACAS 2008. LNCS, vol. 4963, pp. 337-340. Springer, Heidelberg (2008). https://doi.org/10.1007/978-3-540-78800-3_24

16. Del Tedesco, F., Sands, D., Russo, A.: Fault-resilient non-interference. In: Proceedings of Computer Security Foundations Symposium (CSF), pp. 401-416. IEEE (2016) 
17. Eilers, M., Müller, P., Hitz, S.: Modular product programs. In: Ahmed, A. (ed.) ESOP 2018. LNCS, vol. 10801, pp. 502-529. Springer, Cham (2018). https://doi. org/10.1007/978-3-319-89884-1_18

18. Ernst, G., Murray, T.: SecC tool description and Isabelle theories for SecCSL (2019). https://covern.org/secc

19. Goguen, J., Meseguer, J.: Security policies and security models. In: Proceedings of Symposium on Security and Privacy (S\&P), Oakland, California, USA, pp. 11-20, April 1982

20. Gruetter, S., Murray, T.: Short paper: towards information flow reasoning about real-world C code. In: Proceedings of Workshop on Programming Languages and Analysis for Security (PLAS), pp. 43-48. ACM (2017)

21. Gu, R., et al.: CertiKOS: an extensible architecture for building certified concurrent OS kernels. In: Proceedings of USENIX Symposium on Operating Systems Design and Implementation (OSDI), November 2016

22. Jacobs, B., Smans, J., Philippaerts, P., Vogels, F., Penninckx, W., Piessens, F.: VeriFast: a powerful, sound, predictable, fast verifier for $\mathrm{C}$ and java. In: Bobaru, M., Havelund, K., Holzmann, G.J., Joshi, R. (eds.) NFM 2011. LNCS, vol. 6617, pp. 41-55. Springer, Heidelberg (2011). https://doi.org/10.1007/978-3-642-20398$5 \_4$

23. Karbyshev, A., Svendsen, K., Askarov, A., Birkedal, L.: Compositional noninterference for concurrent programs via separation and framing. In: Bauer, L., Küsters, R. (eds.) POST 2018. LNCS, vol. 10804, pp. 53-78. Springer, Cham (2018). https://doi.org/10.1007/978-3-319-89722-6_3

24. Klein, G., et al.: Comprehensive formal verification of an OS microkernel. ACM Trans. Comput. Syst. 32(1), 2:1-2:70 (2014)

25. Leroy, X.: Formal verification of a realistic compiler. Commun. ACM 52(7), 107$115(2009)$

26. Löding, C., Madhusudan, P., Murali, A., Peña, L.: A first order logic with frames. http://madhu.cs.illinois.edu/FOFrameLogic.pdf

27. Lourenço, L., Caires, L.: Dependent information flow types. In: Proceedings of Principles of Programming Languages (POPL), Mumbai, India, pp. 317-328, January 2015

28. Mantel, H., Sands, D.: Controlled declassification based on intransitive noninterference. In: Chin, W.-N. (ed.) APLAS 2004. LNCS, vol. 3302, pp. 129-145. Springer, Heidelberg (2004). https://doi.org/10.1007/978-3-540-30477-7_9

29. Mantel, H., Sands, D., Sudbrock, H.: Assumptions and guarantees for compositional noninterference. In: Proceedings of Computer Security Foundations Symposium (CSF), Cernay-la-Ville, France, pp. 218-232, June 2011

30. Müller, P., Schwerhoff, M., Summers, A.J.: Viper: a verification infrastructure for permission-based reasoning. In: Jobstmann, B., Leino, K.R.M. (eds.) VMCAI 2016. LNCS, vol. 9583, pp. 41-62. Springer, Heidelberg (2016). https://doi.org/10.1007/ 978-3-662-49122-5 2

31. Murray, T.: Short paper: on high-assurance information-flow-secure programming languages. In: Proceedings of Workshop on Programming Languages and Analysis for Security (PLAS), pp. 43-48 (2015)

32. Murray, T., et al.: seL4: from general purpose to a proof of information flow enforcement. In: Proceedings of Symposium on Security and Privacy (S\&P), San Francisco, CA, pp. 415-429, May 2013

33. Murray, T., Sabelfeld, A., Bauer, L.: Special issue on verified information flow security. J. Comput. Secur. 25(4-5), 319-321 (2017) 
34. Murray, T., Sison, R., Engelhardt, K.: COVERN: a logic for compositional verification of information flow control. In: Proceedings of European Symposium on Security and Privacy (EuroS\&P), London, United Kingdom, April 2018

35. Murray, T., Sison, R., Pierzchalski, E., Rizkallah, C.: Compositional verification and refinement of concurrent value-dependent noninterference. In: Proceedings of Computer Security Foundations Symposium (CSF), pp. 417-431, June 2016

36. Newcombe, C., Rath, T., Zhang, F., Munteanu, B., Brooker, M., Deardeuff, M.: How Amazon web services uses formal methods. Commun. ACM 58(4), 66-73 (2015)

37. Nipkow, T., Wenzel, M., Paulson, L.C. (eds.): Isabelle/HOL-A Proof Assistant for Higher-Order Logic. LNCS, vol. 2283. Springer, Heidelberg (2002). https://doi. org/10.1007/3-540-45949-9

38. O'Hearn, P.W.: Resources, concurrency and local reasoning. In: Gardner, P., Yoshida, N. (eds.) CONCUR 2004. LNCS, vol. 3170, pp. 49-67. Springer, Heidelberg (2004). https://doi.org/10.1007/978-3-540-28644-8_4

39. O'Hearn, P.W.: Continuous reasoning: scaling the impact of formal methods. In: Proceedings of Logic in Computer Science (LICS), pp. 13-25. ACM (2018)

40. O'Hearn, P.W., Yang, H., Reynolds, J.C.: Separation and information hiding. ACM Trans. Programm. Lang. Syst. (TOPLAS) 31(3), 11 (2009)

41. Piskac, R., Wies, T., Zufferey, D.: Automating separation logic using SMT. In: Sharygina, N., Veith, H. (eds.) CAV 2013. LNCS, vol. 8044, pp. 773-789. Springer, Heidelberg (2013). https://doi.org/10.1007/978-3-642-39799-8_54

42. Polikarpova, N., Sergey, I.: Structuring the synthesis of heap-manipulating programs. Proc. ACM Program. Lang. 3(POPL), 72 (2019)

43. Prabawa, A., Al Ameen, M.F., Lee, B., Chin, W.-N.: A logical system for modular information flow verification. Verification, Model Checking, and Abstract Interpretation. LNCS, vol. 10747, pp. 430-451. Springer, Cham (2018). https://doi.org/ 10.1007/978-3-319-73721-8_20

44. Reynolds, J.C.: Separation logic: a logic for shared mutable data structures. In: Proceedings of Logic in Computer Science (LICS), pp. 55-74. IEEE (2002)

45. Sabelfeld, A., Sands, D.: Probabilistic noninterference for multi-threaded programs. In: Proceedings of Computer Security Foundations Workshop (CSFW), pp. 200214. IEEE (2000)

46. Vafeiadis, V.: Concurrent separation logic and operational semantics. In: Proceedings of Mathematical Foundations of Programming Semantics (MFPS), pp. 335351 (2011)

47. Vafeiadis, V., Narayan, C.: Relaxed separation logic: a program logic for C11 concurrency. In: Proceedings of Object Oriented Programming Systems Languages \& Applications (OOPSLA), pp. 867-884. ACM (2013)

48. Volpano, D., Smith, G.: Probabilistic noninterference in a concurrent language. J. Comput. Secur. 7(2,3), 231-253 (1999)

49. Yang, H.: Relational separation logic. Theor. Comput. Sci. 375(1-3), 308-334 (2007)

50. Zheng, L., Myers, A.C.: Dynamic security labels and static information flow control. Int. J. Inf. Secur. 6(2-3), 67-84 (2007) 
Open Access This chapter is licensed under the terms of the Creative Commons Attribution 4.0 International License (http://creativecommons.org/licenses/by/4.0/), which permits use, sharing, adaptation, distribution and reproduction in any medium or format, as long as you give appropriate credit to the original author(s) and the source, provide a link to the Creative Commons license and indicate if changes were made.

The images or other third party material in this chapter are included in the chapter's Creative Commons license, unless indicated otherwise in a credit line to the material. If material is not included in the chapter's Creative Commons license and your intended use is not permitted by statutory regulation or exceeds the permitted use, you will need to obtain permission directly from the copyright holder. 